\title{
BANDS IN PERVASIVE PRE-RIESZ SPACES
}

\author{
O. VAN GAANS AND A. KALAUCH
}

\begin{abstract}
Pre-Riesz spaces are partially ordered vector spaces which are order dense subspaces of vector lattices. A band in a pre-Riesz space can be extended to a band in the ambient vector lattice. The corresponding restriction property does not hold in general. We provide sufficient conditions on the underlying space such that the restriction property for bands holds. As an application, we consider the space $L^{r}\left(l_{0}^{\infty}\right)$ of all regular operators on the space $l_{0}^{\infty}$ of all finally constant sequences. We establish that $L^{r}\left(l_{0}^{\infty}\right)$ is pre-Riesz and that its subspace of all order continuous operators is a band in $L^{r}\left(l_{0}^{\infty}\right)$.
\end{abstract}

Mathematics subject classification (2000): 46A40, 06F20.

Key words and phrases: Band, disjointness, order dense subspace, partially ordered vector space, pre-Riesz space, regular operator, Riesz completion, vector lattice.

\section{REFERENCES}

[1] Y. A. AbRamovich AND G. SirotKin, On order convergence of nets, Positivity 9 (3) (2005), 287-292.

[2] Y. A. ABRAMOVICH AND A. W. WiCKSTEAD, Regular operators from and into a small Riesz space, Indag. Mathem., N.S. 2 (3) (1991), 257-274.

[3] C. D. Aliprantis And O. BuRkinshaw, Positive operators, Academic Press Inc., London, 1985.

[4] G. Buskes AND A. C. M. VAN RoOIJ, The vector lattice cover of certain partially ordered groups, J. Austral. Math. Soc. (Series A) 54 (1993), 352-367.

[5] O. van GaAns And A. Kalauch, Disjointness in partially ordered vector spaces, Positivity 10 (3) (2006), 573-589.

[6] O. VAN GAANS AND A. KALAUCH, Ideals and bands in pre-Riesz spaces, Positivity, to appear.

[7] M. van HaAndel, Completions in Riesz space theory, Ph. D. thesis, University of Nijmegen, 1993. 